\title{
Sgoldstino searches at CERN Electron Beam Dump
}

\author{
Konstantin Astapov ${ }^{1, a}$ \\ ${ }^{1}$ Institute for Nuclear Research of the Russian Academy of Sciences, Moscow 117312, Russia
}

\begin{abstract}
In the present paper we discussed light scalar sgoldstino in context of low scale SUSY breaking. We analyzed electromagnetic sector of interaction of sgoldstino with SM. We also took into account the impact on this interaction of the admixture of lightest MSSM Higgs scalar in the sgoldstino state. The signal rate of rare sgoldstino decays into SM particles at CERN electron beam dump experiment NA64 for the scalar sgoldstino has been estimated. We used expected signal rate to obtain some regions of sgoldstino parameter space (sgoldstino mass and SUSY breaking scale $\sqrt{F}$ ).
\end{abstract}

\section{Introduction}

Supersymmetry is a promising extention of the Standard Model [1, 2]. However according to experimentally observed absence of superpartners at low energies, SUSY models imply supersymmetry to be spontaneously broken at some scale. The breaking mechanism is provided by underlying microscopic theory. The breaking can happen when a hidden sector dynamics results in a nonzero vacuum expectation value $F$ to an auxiliary component of the superfield [3] and $\sqrt{F}$ is SUSY breaking scale. According to supersymmetric analog of the Goldstone theorem [4] there should exist a massless fermionic degree of freedom, goldstino.

In the simplest case this fermion belongs to a chiral multiplet, dubbed goldstino supermultiplet. Apart from goldstino, it contains scalar - sgoldstino and an auxiliary field acquiring nonzero vacuum expectation value $F$, which trigger spontaneous SUSY breaking. The quantity $\sqrt{F}$ is supersymmetry breaking scale. Couplings of sgoldstino to SM fields are suppressed by $\frac{1}{F}$ and expected to be quite small. Being included into supergravity framework goldstino becomes longitudinal component of gravitino with mass related to the scale of supersymmetry breaking $\sqrt{F}$ as follows $m_{3 / 2}=\frac{F}{\sqrt{3} M_{p l}}$, where $M_{p l}$ is the Planck mass [5]. In the present work we consider the mass of sgoldstino to be light (less than $1 \mathrm{GeV}$ ) as phenomenologically interesting case; therefore a high intensity beam is required to test the model via production of sgoldstino. Beam dump experiment can perform the task. A preliminary estimate of the search for sgoldstino at beam dump experiment SHiP for sgoldstino production and decay mechanisms can be found in Ref. [6]; here we complete that study by considering production of sgoldstino in electron-proton collisions.

The purpose of the present work is to estimate the signal rate of sgoldstino decays expected to detect at the NA64 electron beam-dump experiment in context of present model (see Refs. [7-9] for description of the experiment in context of the search for Dark photon). We consider the case when mostly the sgoldstino decays into electron-positron pairs as searching signature.

\footnotetext{
ae-mail: astapov@inr.ac.ru
} 


\section{Model and parameters}

To the leading order in $1 / F$, sgoldstino couplings to SM gauge fields - photons $F_{\mu \nu}$, gluons $G_{\mu \nu}$ and matter fields - leptons $l_{a}$, up and down quarks $u_{a}$ and $d_{a}$, where index $a$ runs over three gererations at the mass scale above $\Lambda_{Q C D}$ but below electroweak symmetry breaking reads as $[10,11]$

$$
\begin{aligned}
\mathcal{L}_{e f f}^{s}=-\frac{M_{\gamma \gamma}}{2 \sqrt{2} F} s F^{\mu v} F_{\mu \nu}-\frac{M_{2}}{\sqrt{2} F} s W^{\mu v} W_{\mu \nu}-\frac{M_{Z Z}}{2 \sqrt{2} F} s Z^{\mu v} Z_{\mu \nu}-\frac{M_{3}}{2 \sqrt{2} F} s \operatorname{Tr} G^{\mu v} G_{\mu \nu}- & \\
- & -\frac{A_{a b}^{U} v}{\sqrt{2} F} s u_{a} u_{b}-\frac{A_{a b}^{D} v}{\sqrt{2} F} s d_{a} d_{b}--\frac{A_{a b}^{L} v}{\sqrt{2} F} s l_{a} l_{b}
\end{aligned}
$$

Here $M_{3}$ is the gluino mass, $M_{\gamma \gamma}=M_{1} \sin ^{2} \theta_{W}+M_{2} \cos ^{2} \theta_{W}$ and $M_{Z Z}=M_{1} \cos ^{2} \theta_{W}+M_{2} \sin ^{2} \theta_{W}$ with $M_{1}$ and $M_{2}$ being $U(1)_{Y^{-}}$and $S U(2)_{W^{-}}$-gaugino masses and $\theta_{W}$ the weak mixing angle, and $A_{a b}^{U}, A_{a b}^{D}$ and $A_{a b}^{L}$ are soft trilinear coupling constants. Lagrangian (1) includes only single-sgoldstino interaction terms; considered in Refs. [11-14], double-sgoldstino terms are suppressed by $1 / F^{2}$ and are not probable for testing at the NA64 experiment.

In general sgoldstino also mixes with neutral Higgs bosons as discussed in Refs. [10, 15-17]: the scalar sgoldstino $S$ mixes with neutral light $h$ and heavy $H$ Higgs bosons, while pseudoscalar $P$ mixes with their axial partner $A$. We account for the mixing with $h$ only, since the other two do not change light scalar sgoldstino phenomenology at NA64 for considered set of parameters of the model. Mixing of the scalar sgoldstino and the lightest MSSM Higgs boson (SM-like Higgs) has been calculated in [10].

Here we are considering sgoldstino $S$ mass to be less than $1 \mathrm{GeV}$ (much lighter than the SM-like Higgs boson of mass $m_{h} \approx 125 \mathrm{GeV}$ ) then the mixing has negligible impact on Higgs mass.

In Table 1 we set numerical values for parameters of the MSSM so that $h$ asquire its experimentally observed value of $125 \mathrm{GeV}$ by loop corrections from squark masses and trilinear couplings; and $H$ along with $A$ fields acquire heavy masses over $1 \mathrm{TeV}$ to not to contribute into mixing with scalar and pseudoscalar sgoldstinos. In this arbitrary choice we suppose that all the model parameters take experimentally allowed values.

\begin{tabular}{llllllll}
\hline$M_{1}, \mathrm{GeV}$ & $M_{2}, \mathrm{GeV}$ & $M_{3}, \mathrm{GeV}$ & $\mu, \mathrm{GeV}$ & $\tan \beta$ & $m_{A}, \mathrm{GeV}$ & $A_{l}, \mathrm{GeV}$ & $A_{Q}, \mathrm{GeV}$ \\
\hline 100 & 250 & 1500 & 1000 & 6 & 1000 & 2800 & 0 \\
\hline
\end{tabular}

Table 1. MSSM benchmark point.

In the table we denoted $A_{a a}^{U}, A_{a a}^{D}$ as $A_{Q}$ and $A_{a a}^{L}$ as $A_{l}$, all the off-diagonal $A_{a b}^{U, D, L}$ are set to zero.

\section{Results}

Here we estimate the number of $e^{-} N \rightarrow S \rightarrow e^{+} e^{-}$events inside the fiducial volume of the NA64 experimental setup. Experimental setup is designed to search for rare decays with charged particles in the final state. Detailed setup scheme is outlined in the Ref.[7]. The experiment utilize clean high energy $e^{-}$beam with less then $10^{-2}$ level of impurities and momenta of $100 \mathrm{GeV}$. Electron beam is produced by primary $400 \mathrm{GeV}$ proton beam from SPS hitting the primary beryllium target. Target calorimeter thickness is $l_{s h}=0.15 \mathrm{~m}$. The vacuum vessel length is about $l_{\text {det }}=15 \mathrm{~m}$. It forms a cylinder along the beam axis with an circle base of $30 \mathrm{~cm}$ in diameter. 
Electron beam strikes on the electron calorimeter target and produces sgoldstinos directly. With chosen set of parameters of the model only electromagnatic interaction involved in scalar sgoldstino production in electron-proton collisions of electron beam hitting heavy nuclei lead $(Z=82)$ target. Then we take into account interactions of sgoldstino with nuclei and electrons in calculation of the production cross-section.

At the back end of the vacuum vessel another electronic calorimeter serves for count of electromagnetic shower produced by subsequent sgoldstino decays into charged particles. For (sub-)GeV mass-range sgoldstino decay channels into pairs of SM particles, if kinematically allowed are: $\gamma \gamma$, $e^{+} e^{-}, \mu^{+} \mu^{-}, \pi^{0} \pi^{0}, \pi^{+} \pi^{-}$.

Using sgoldstino production cross-section and it's decay width into pairs of charged particles we can obtain the number of signal events. It reads as

$$
N_{\text {signal }}=N_{\mathrm{EOT}} \frac{N_{0} X_{0}}{A} \int_{m_{S}}^{E_{0}-m_{e}} d E_{S} \int_{E_{S}+m_{e}}^{E_{0}} d E_{e} \int_{0}^{T} d t\left[I_{e}\left(E_{0}, E_{e}, t\right) \frac{1}{E_{e}} \frac{d \sigma}{d x_{e}}\right] w_{d e t} \mathrm{BR}_{d e t},
$$

where the expected number of electons on the target is $N_{\mathrm{EOT}}=10^{9}, N_{0}$ is Avagadro's number, $X_{0}$ is the unit radiation length of the target material, $\mathrm{A}$ is atomic mass number, $E_{s}$ is sgoldstino energy, $E_{e}$ is initial electron energy and $w_{d e t}$ denotes the probability for the sgoldstino to decay inside the fiducial volume of the detector,

$$
w_{\text {det }}\left(E_{S(P)}, m_{S(P)}, \sqrt{F}\right)=\exp \left(-l_{s h} / \gamma c \tau_{S(P)}\right) \times\left[1-\exp \left(-l_{\text {det }} / \gamma c \tau_{S(P)}\right)\right],
$$

with the sgoldstino gamma factor $\gamma=E_{S(P)} / m_{S(P)}$.

Since electron beam with energy $E_{0}$ becomes degraded as electrons pass trough and interact with its nucleus. Energy distribution of electrons (see Ref.[18]) after passing through material by $t$ radiation length is given by:

$$
I_{e}\left(E_{0}, E_{e}, t\right)=\frac{1}{E_{0}} \frac{\left[\ln \left(\frac{E_{0}}{E_{e}}\right)\right]^{b t-1}}{\Gamma(b t)},
$$

where $\Gamma$ is Gamma function, $b=4 / 3, E_{0}$ is initial beam energy at $t=0$.

In Fig. 1 we indicate the region in the model parameter space $\left(m_{S}, 1 / \sqrt{F}\right)$, where the number of sgoldstino decay events inside the fiducial volume exceeds $3, N_{\text {signal }}>3$. That is, if no events were observed the region is excluded at the confidence level of 95\%, in accordance with the Poisson statistics. The lower boundary in Fig. 1 is the region where the couplings are so small that sgoldstinos escape from the detector without decay. The upper boundary corresponds to case when couplings are so large that sgoldstinos decay before the detector. The scalings of the signal events imply that models with a higher (as compared to that presented in Fig. 1) scale of supersymmetry breaking can be tested if MSSM parameters $\mu, M_{\gamma \gamma}$ are appropriately larger (as compared to those presented in Table 1).

\section{Conclusions}

We have estimated sensitivity of the NA64 experiment to supersymmetric extensions of the SM where sgoldstinos are light. The experiment will be able to probe the supersymmetry breaking scale $\sqrt{F}$ up to $10^{4} \mathrm{TeV}$. We have obtained exclusion regions of the scalar sgoldstino parameter space $\left(m_{S}\right.$ vs. $1 / \sqrt{F})$.

\section{Acknowledgments}

We thank D. Kirpichnikov, D. Gorbunov, S. Demidov, S. Gninenko, M. Kirsanov, N. Krasnikov and S. Kulagin for valuable discussions. The work was supported by the RSF Grant No. 14-22-00161. 


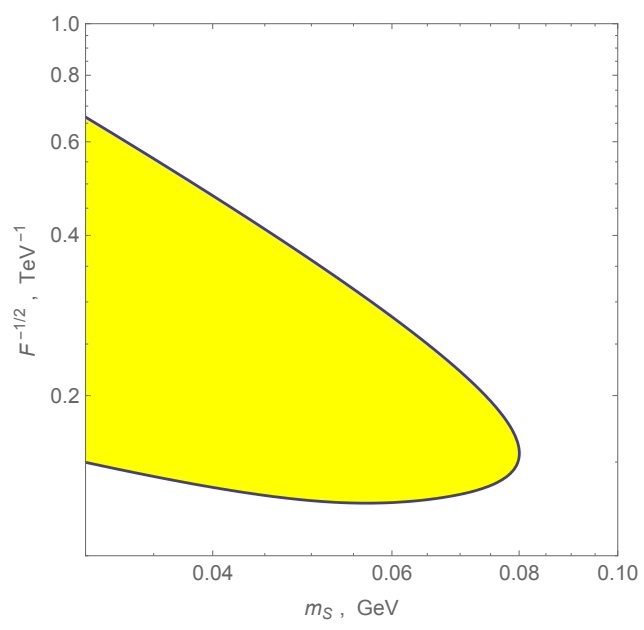

Figure 1. The shaded region will be probed at the NA64 experiment.

\section{References}

[1] H. E. Haber and G. L. Kane, "The Search for Supersymmetry: Probing physics beyond the standard model," Phys. Rep. 117, 75 (1985).

[2] S. P. Martin, "A Supersymmetry primer, In *Kane, G.L. (ed.): Perspectives on supersymmetry II* 1-153 [hep-ph/9709356].

[3] A. Brignole, J. A. Casas, J. R. Espinosa and I. Navarro, Nucl. Phys. B 666, 105 (2003) doi:10.1016/S0550-3213(03)00539-X [hep-ph/0301121].

[4] D. V. Volkov and V. P. Akulov, JETP Lett. 16, 438 (1972) [Pisma Zh. Eksp. Teor. Fiz. 16, 621 (1972)], D. V. Volkov and V. P. Akulov, Phys. Lett. 46B, 109 (1973).

[5] E. Cremmer, B. Julia, J. Scherk, P. van Nieuwenhuizen, S. Ferrara and L. Girardello, Phys. Lett. 79B, 231 (1978). doi:10.1016/0370-2693(78)90230-7

[6] K. O. Astapov and D. S. Gorbunov, Phys. Rev. D 93, no. 3, 035008 (2016) doi:10.1103/PhysRevD.93.035008 [arXiv:1511.05403 [hep-ph]].

[7] S. Andreas et al., arXiv:1312.3309 [hep-ex].

[8] D. Banerjee et al. [NA64 Collaboration], arXiv:1610.02988 [hep-ex].

[9] S. N. Gninenko, N. V. Krasnikov, M. M. Kirsanov and D. V. Kirpichnikov, Phys. Rev. D 94, no. 9, 095025 (2016) doi:10.1103/PhysRevD.94.095025 [arXiv:1604.08432 [hep-ph]].

[10] K. O. Astapov and S. V. Demidov, JHEP 1501, 136 (2015) doi:10.1007/JHEP01(2015)136 [arXiv:1411.6222 [hep-ph]].

[11] D. S. Gorbunov, "Light sgoldstino: Precision measurements versus collider searches," Nucl. Phys. B 602, 213 (2001) [hep-ph/0007325].

[12] E. Perazzi, G. Ridolfi and F. Zwirner, "Signatures of massive sgoldstinos at e+ e- colliders," Nucl. Phys. B 574, 3 (2000) [hep-ph/0001025].

[13] E. Perazzi, G. Ridolfi and F. Zwirner, "Signatures of massive sgoldstinos at hadron colliders," Nucl. Phys. B 590, 287 (2000) [hep-ph/0005076].

[14] S. V. Demidov and D. S. Gorbunov, "Flavor violating processes with sgoldstino pair production," Phys. Rev. D 85, 077701 (2012) [arXiv:1112.5230 [hep-ph]]. 
[15] E. Dudas, C. Petersson and P. Tziveloglou, "Low Scale Supersymmetry Breaking and its LHC Signatures,” Nucl. Phys. B 870, 353 (2013) [arXiv:1211.5609 [hep-ph]].

[16] B. Bellazzini, C. Petersson and R. Torre, "Photophilic Higgs from sgoldstino mixing," Phys. Rev. D 86, 033016 (2012) [arXiv:1207.0803 [hep-ph]].

[17] S. V. Demidov and I. V. Sobolev, JHEP 1608, 030 (2016) doi:10.1007/JHEP08(2016)030 [arXiv:1605.08220 [hep-ph]].

[18] S. Andreas, C. Niebuhr and A. Ringwald, Phys. Rev. D 86, 095019 (2012) doi:10.1103/PhysRevD.86.095019 [arXiv:1209.6083 [hep-ph]]. 